\title{
Representing Model Differences by Delta Operations
}

\author{
Dilshodbek Kuryazov, Andreas Winter \\ Software Engineering Group \\ University of Oldenburg \\ \{kuryazov,winter\}@se.uni-oldenburg.de
}

\begin{abstract}
Software models are subject to subsequent changes. Models evolve over time to meet changes resulting in several versions. The differences between subsequent model versions are represented in differences documents usually referred to as Modeling Deltas. The model differences are used in establishing several further services and operations of version control, collaborative modeling and history analysis tools for software models. Therefore, finding an adequate difference representation approach is essential for applicability, re-usability and analysis of the model differences.
\end{abstract}

This paper introduces the Delta Operation Language (DOL), a meta-model independent and operation-based concept to model difference representations. The proposed approach provides several operative $D O L$-services for utilizing the DOL-based differences. The application areas of DOL include Model Versioning, Model History Analysis and Collaborative Modeling. The approach is applied to UML activity diagrams as a running example.

\section{Motivation}

Software models are widely used in designing software systems on different abstraction levels. Like code of software projects, software models evolve over time undergoing various changes such as extensions, improvements and corrections. Constantly changing a model results in different versions of a model during its lifetime. The consistent recognition, specification and exploitation of the differences between subsequent model versions are crucial in comprehending, analysing and managing model-based software systems.

For dealing with modifications on models, the differences between model versions have to be properly identified and represented in difference documents i.e. Modeling Deltas. Representation of the model differences in modeling deltas is an important concern for version control, collaborative modeling and history analysis tools.

Model versioning systems used to handle and store the histories of evolving software models. They usually take advantage of modeling deltas to store the model differences. Each modeling delta represents the differences between subsequent versions of a model. A model versioning system reduces the difference storage space and improves simplicity by storing only deltas.

Changes are applied to software models by a group of designers using collaborative modeling tools. In the case of collaborative modeling, exchanging the model differences is eased by exchanging only modeling deltas which contain only the differences. Exchanging small deltas enables them to synchronize changes rapidly by reducing the capacity of exchange data.

Analysing the model histories helps to understand the evolutionary life-cycle of models. Information about the model histories is gathered by tracking the change histories of each model element. As long as only changed model elements are referred to in modeling deltas, tracing the change history from modeling deltas help to rapidly detect required history information. Afterwards, the users can browse or visualize that information to see how a model evolves.

Software models do not follow the similar principles and syntax as code-based projects. Therefore, the rich data and compound structure of models has be considered in representation of the model differences. Difference representation has to be capable of fully handling and carrying all necessary information about the change histories. Additionally, the differences represented in modeling deltas must be easy to access for further re-use and manipulations. Software models also can be represented as textual files using exchange formats like XMI (XML Metadata Interchange ) [1]. But, it is commonly admitted that differentiating textual representation of models does not follow modeling concepts [2], [3]. The code-centric versioning systems like Git [4], Subversion [5] or RCS [6] can not fully handle software models in exchange formats and do not produce useful information about the model differences. To this end, this paper addresses the problem of difference representation for software models.

This paper introduces the general Delta Operations Language (DOL) of Generic Model Versioning System (GMoVerS) to model difference representations. DOL is a set of domain specific languages to model difference representation in terms of delta language operations. In order to derive a specific DOL for a specific modeling language, the meta-model of a modeling language is required. A DOL Generator used to generate a specific DOL for a certain modeling language using the meta-model. Then, a specific DOL is fully capable of representing all differences between subsequent versions of the instance models in terms of operation-based DOL in modeling deltas. The operations in modeling deltas are referred to as Delta Operations.

Moreover, DOL aims at supporting several DOL-services which can access and reuse delta operations. These operative services make the DOL-based modeling deltas quite handy in various application areas and enable application areas to utilize the DOL-based modeling deltas. Operation-based DOL has several use cases in this paper. These are Generic Model Versioning System (GMoVerS) (discussed in Section VIII-A), Model History Analysis (Section VIII-B), Collaborative Modeling (Section VIII-C) which is in process.

The paper is structured as follows: Section II lists requirements that difference representation has to satisfy. Several related approaches are discussed in Section III. Section IV gives an example of an evolving model and DOL-based difference 
representation between subsequent model versions. Section V explains DOL generation from the given meta-model. The DOL-services are introduced in Section VI. The prototype implementations are explained in Section VII. Insight into application areas of DOL is given in Section VIII. The paper ends up by drawing conclusions and outlook for future work in Section IX.

\section{REQUIREMENTS}

In the case of software models, difference representation has to satisfy a number of requirements regarding its reusability, applicability and optimality. Difference information (i.e. history information of model changes) must be small and complete with a simple syntax and re-usable when needed. Besides, the difference representation approach has to be independent from modeling languages and modeling tools enabling applicability. Consequently, in order to have a solid difference representation technique in the end, several requirements and properties following concrete principles are defined targeting re-usability and applicability of modeling deltas and efficient access of model versions:

- Meta-model Generic. There are several modeling languages which follow different formal specifications and concepts. The abstract syntax of modeling languages i.e. the modeling concepts are defined by the languages meta-model. Models conforming that modeling language are subject to continuous changes and evolution. Thus, being meta-model independent makes a difference representation approach applicable to all modeling languages with respect to their metamodels.

- Tool Independent. There are several modeling tools and they have own internal model representations. To be able to handle models designed in different modeling tools, difference representation must not rely on an internal model representation of a specific model designing tool restricting itself for that modeling tool.

- Operation-based. The model differences are the collection of changes of modeling concepts. Changes of modeling artefacts can be viewed as operations that are applied to a previous version to form the later. These operations have to allow for representing all model changes embodying all necessary information about the change histories. Additionally, using an operation for each separate change results in a low number of operations with simple syntax and allows effortless implementations as well as follows actual modeling concepts. These operations have to be composite to enable representation of complex model changes.

- Delta-based. After representing changes in terms of operations, a certain set of operations between two states of a model should be grouped and stored in a modeling delta. A modeling delta consists of a set of operations which refer only to changed modeling concepts, meanwhile that delta performs the executable descriptions of the differences which allows to revert older model versions.

- Completeness. The representation must carry precise information about each change including the kind of change and the referenced modeling concept. The kind of change defines what kind of change is made (cf. creation or deletion of an object or change of object attributes), whereas the referenced modeling concept admits the changed model object.

- Reusability. The model differences represented in modeling deltas have to be available for further reuse and exploitation. Only representing the model differences without being accessible and re-usable is ineffective and needless. Thus, difference representation must be straightforward and accessible fur further analysis and manipulations enabling applicability to various application areas.

These significant principles are also design foundation of the DOL-based representation approach that contribute to empower the qualification and solidity of difference representation. The approach aims at fulfilling these requirements throughout this paper.

\section{RELATED WORK}

Before explaining operation-based DOL in detail, this section discusses several existing difference representation approaches. Also, these approaches are reviewed in the context of principles listed in Section II.

An early approach on operation-based difference representation is introduced by Alanen and Porres [7] which is metamodel independent as well. The approach addresses calculation of the differences and union of models. It represents the differences as a sequence of difference operations. Moreover, a number of merge conflicts are extended by the combination of the difference operations. The approach is meta-model generic, operation-based and provides a conflict resolution technique.

A meta-model independent approach for difference representation is introduced by Cicchetti et al. [2], [8]. The approach uses a differences model for representing the model differences. The differences model conforms to the differences meta-model derived from the base meta-model by automatic transformations. The approach provides a difference application component which requires model matching between the differences and base models to detect weaving model. The weaving model is used to contain references between the differences and base model elements. Conflict resolution is provided to detect and resolve conflicts while merging the differences and base models. Cicchetti et al. is metamodel generic, provides difference application and conflict resolution services, but it uses model-based way of difference representation.

SMOVER (Semantically enhanced Model Version Control System) [9] is a state-based approach, which provides several standard versioning activities such as add - to bring new models under version control, checkout - to obtain a copy of a model in the central repository into the local working space, commit - to commit local changes to the central repository and update - to update the local working space with the latest changes in the central repository. SMOVER mostly addresses flexible difference merging technique and uses standard SQL databases to store the model differences. Hence, the approach requires to have an adapter which lies between external modeling tools and SMOVER. 
Haber et al. [10] addresses engineering delta modeling languages for software product lines. The approach aims at automatically deriving delta operations for software architectures of software product lines. But, it relies on text-based models and requires to specify the grammar of a modeling language to derive a delta language. The approach is operation-based, but focuses on software product lines.

An approach for specifying and recognizing model changes based on edit operations (MOCA) is introduced by Kehrer et al. [11], [12]. The approach consists of a chain of model differencing steps including model matching, deriving lowlevel changes and semantic lifting phases for EMF (Eclipse Modeling Framework) [3] models. The detected low-level changes are grouped and shifted up to a high-level syntax using the "semantic lifting" component. The authors define the set of recognition rules to recognize low-level changes and produce the user-level changes. These set of recognition rules cover 41 different edit operations for UML class diagrams [13] and 16 different edit operations for Matlab/Simulink models [14]. The resulting user/high-level changes are represented in models with different colours. MOCA is operation-based, uses modelbased way for representations and focuses on EMF models.

Koegel and Helming [15] developed the EMF Store framework which is a data model repository for EMF models [3]. The framework allows collaborative work of several modellers directly via peer-to-peer connection providing semantic versioning of models, branching and conflict detection while merging. The EMF Store platform is extended by Krusche and Bruegge with Model-based Real-time Synchronization [16]. The EMF Store uses operation-based change tracking considering an atomic change of the instance models or a composite of several atomic changes. EMF Store is operationbased, re-usable, delta-based, provides both state-based and runtime operation recording but addresses EMF models.

Another model-based difference representation approach, EMF compare and merge [3] was introduced for differencing EMF models. The EMF compare provides model comparison and merging services and relies on EMF models.

These approaches already provide a support to deal with some aspects and principles of model difference representation. All the existing approaches provide a form of difference representation even some of them are strict to a specific modeling language, but most of them lack providing sufficient catalogue of additional services to manipulate their difference information. GMoVerS DOL aims at supplying a wide range of the DOL-services which can directly access and reuse the DOL-based repositories. Besides, the approach in this paper satisfies all the principles in Section II which are literally important for applicability and exploitation of model difference representations.

\section{EXAMPLE}

This section covers a simple example of model difference representation by the GMoVerS DOL. To express the idea behind the approach, a simplified UML activity diagram [13] is used as running example throughout this paper.

Figure 1 portrays a simplified meta-model of UML activity diagram. The current prototype implementation of the
DOL Generator imports meta-models designed as UML Class diagrams. DOL is derived for this specific meta-model to explain the example in this section, but DOL Generation is completely independent from meta-models satisfying the Meta-model Genericness principle.

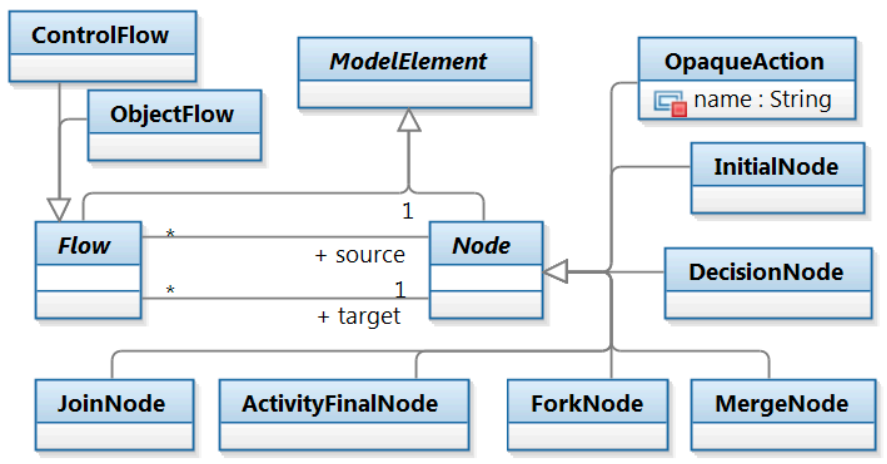

Figure 1: Simplified UML Activity Diagram meta-model

All meta-model classes are either Nodes or Flows (each flow having source and target attributes) and both are of type model ModelElement. Only Action has the name attribute whereas the other classes have no attributes.

Figure 2 depicts three consecutive versions of the same UML activity model performing an Ordering System example. All model versions conform to the same simplified meta-model shown in Figure 1.
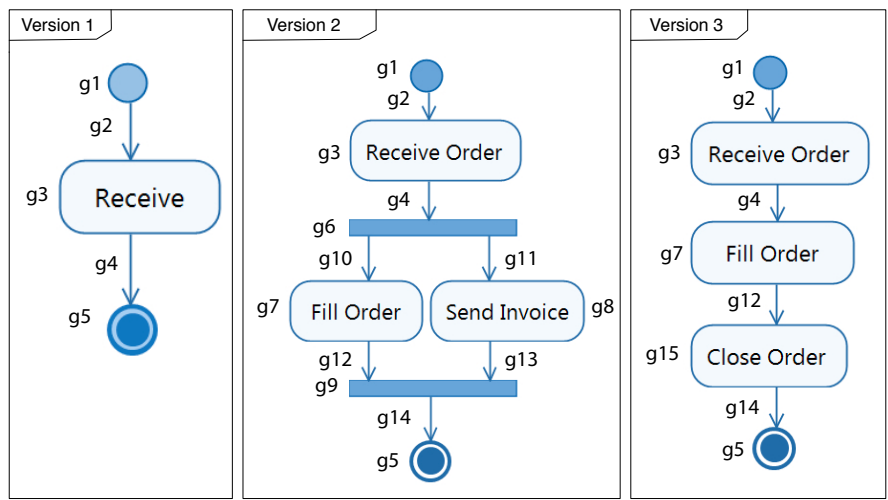

Figure 2: Example activity diagram in three concurrent versions

Each model element is assigned to a certain persistent identifier $\left(g_{x}\right)$. The first version contains an Action named Receive as well as an Initial and a Final node. All elements are connected by (Control) Flows. The model evolves into the second version after several modifications such as Fork, Join nodes, two Actions named Fill Order and Send Invoice are created, the target end of Control Flow $\mathrm{g} 4$ is reconnected to the fork node, the name of the Receive action 93 is changed, and several control flows are created connecting these nodes. Finally, the model evolves again into the third version after creating a new action with name Close Order, reconnecting the target ends of $g 4$ and $g 12$, and the source end of 914 and deleting Fork and Join-nodes, the Send Invoice action, and the control flows g10, g11 and g13.

To represent these differences, the specific DOL for UML activity diagrams is derived from its meta-model in Figure 
In Figure 6, all model elements including flows and nodes can be deleted, also, they can be created with parameters if they have attributes. Only attributes can be changed and all associations are associated with attributes. Attributes are created when their composing elements are created and deleted when their composing elements are deleted.

The specific DOL is generated as Java Interface by a $D O L$ Generator (Figure 8) importing the meta-model of a modeling language. The methods of the resulting interface are named and parametrized according to the meta-model concepts including one of the create, delete and change operations (Figure 7). Each implementation of these methods results in an analogous operation with relevant parameters.

Considering the activity diagram example presented in Section IV, the DOL Generator imports the simplified metamodel depicted in Figure 1.

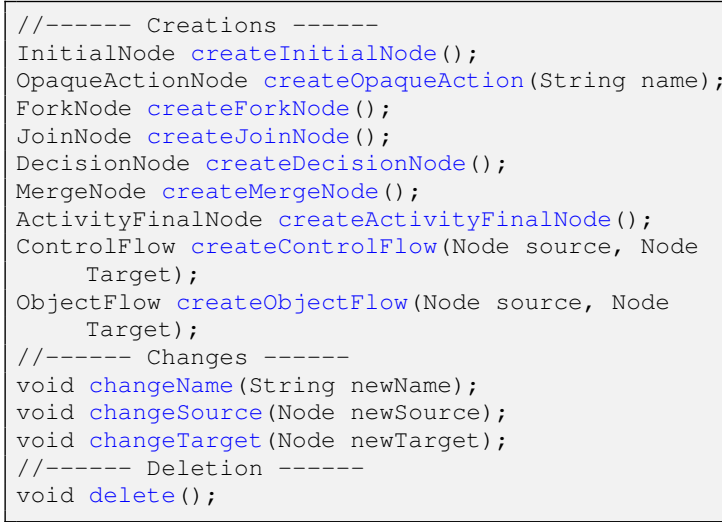

Figure 7: Interface generated from UML Activity Diagram meta-model

In case of the meta-model of any other modeling language, the DOL Generator follows the same principle to generate DOL i.e. only a meta-model is imported and three basic operations are applied to each concept of that meta-model. Eventually, each of the interface methods has the same structure as a Do part and an Object part. The delta operations are produced by implementations of that interface by assigning persistent identifiers.

\section{DOL SERVICES}

Representing the model differences by modeling deltas is not only focus of GMoVerS DOL. Besides, this paper introduces a reasonable set of $D O L$-services enabling reusability and applicability of the DOL-based deltas. Figure 8 displays the overall architecture of the DOL-services and the potential orchestration of them based on data-flows among these services.

The amalgamation depicts the DOL-services such as an Adapter, a Difference Calculator, a Delta Optimizer, a Patcher and a Change Tracer. Each DOL-service has a particular task and is involved in constructing the specific orchestration in the framework of DOL applications. For instance, models designed in external modeling tools are imported into the system through the Adapter. The adapter parses models in the exchange formats to internal representations and vice versa.
The difference calculator is used to detect the differences between the compared models and produce the differences and active deltas by implementing a DOL interface. The difference calculator uses the Optimizer to produce the optimized modeling deltas and to write them into a repository. After all, other DOL-services such as the Patcher and the Change Tracer are capable of utilizing the DOL operations. The patcher is used to revert older model versions. The change Tracer tracks a specified model element and reports the change history of that element. Then, these change reports can be visualized to analyse the change history. All of these DOL-services are discussed in detail in the following sections.

These DOL-services are employed in developing the DOL applications such as Model Versioning, Collaborative Modeling and Model History Analysis (discussed in Section VIII). Architectures of these applications are built based on the specific orchestrations of several DOL-services in a certain order.

\section{A. Adapter}

Software models are created by using model designing tools and mostly these tools do not provide proper model versioning, collaborative modeling or model history analysis support. Most commercial modeling tools such as Visual Paradigm (VP) [17], Rational Software Architect (RSA) [18] or open source tool Eclipse Modeling Framework (EMF) [3] have their own internal model representation techniques. Thus, integrating version management or collaborative modeling tools with model designing tools is a challenge. But, these tools provide export and import of software models by XML Metadata Interchange (XMI) [1] format without layout information. Therefore, to exchange models between the external modeling tools and the GMoVerS version control system, the DOL-services provide the Adapter (Figure 9).

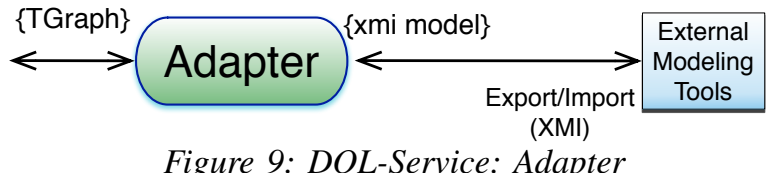

In GMoVerS, models are represented as TGraphs internally [19] (discussed in Section VII) to make them generally processable in implementation of the DOL applications. The Adapter can parse models in both directions: from the exchange formats to TGraphs and vice versa. The adapter also uses the meta-model of a modeling language to recognize all modeling concepts specified in the exchange format. Therefore, it is independent from model designing tools, modeling languages and exchange format versions in regard to a metamodel.

\section{B. Difference Calculator}

The difference calculator detects the differences between compared models using state-based comparison and produces the delta operations. A number of approaches already exist for difference calculation. Therefore, Küpker [20] investigated existing approaches such as UMLDiff [21] and SiDiff [22], [23] and combined them to gDiff (generic differentiating) tool. 


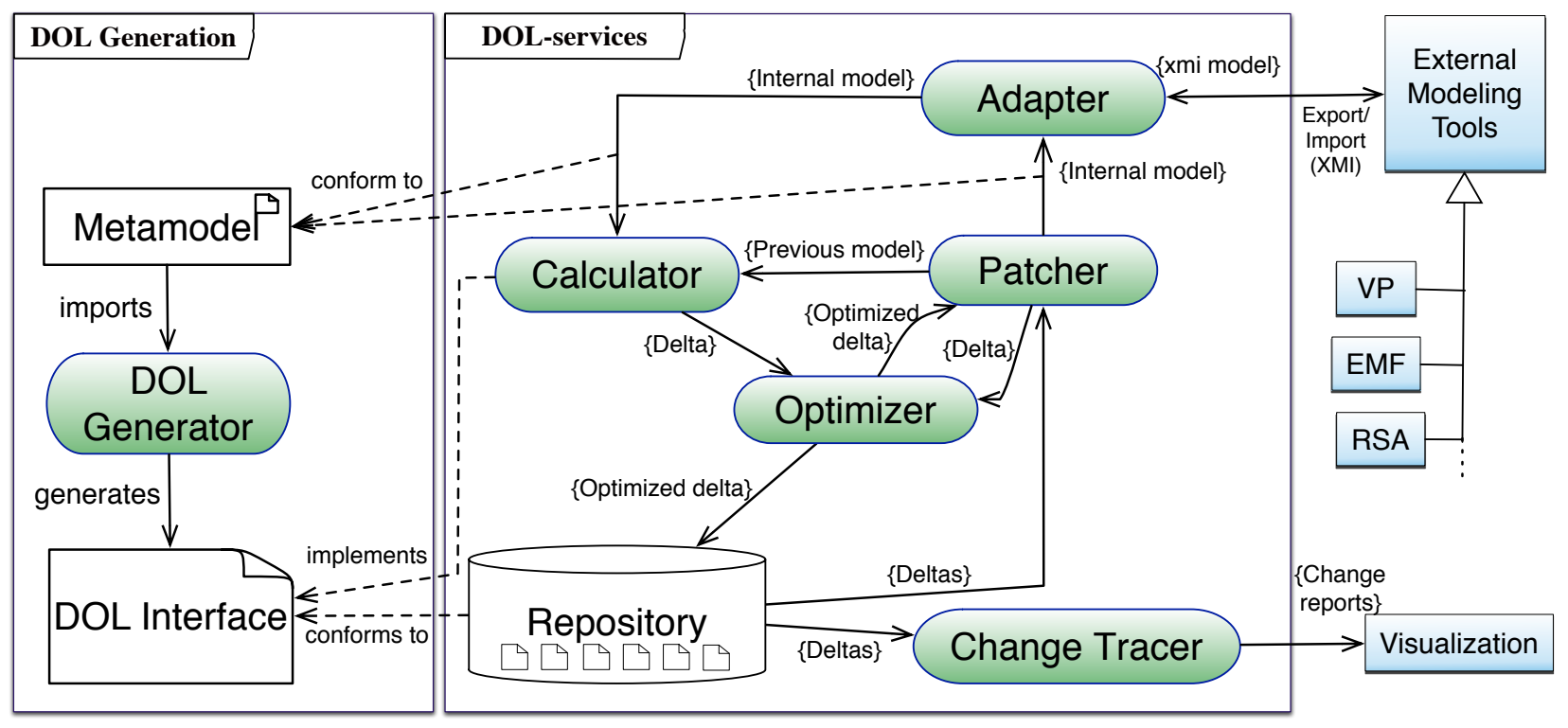

Figure 8: DOL generation and potential orchestration of the DOL-services

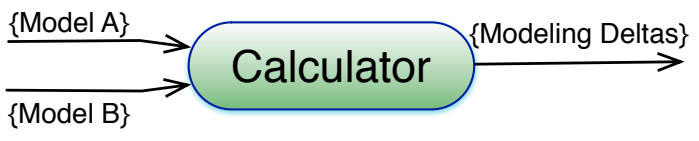

Figure 10: DOL-Service: Difference calculator

The calculator used in GMoVerS firstly detects possible matches between two compared models (Model A and Model $\mathrm{B}$ in Figure 10), and then calculates the model similarities using similarity metrics such as a name [21] or a structural similarity [23]. The candidate element with the highest similarity is selected as an unique match for the host element and created, deleted and changed elements are detected.

After detecting such modifications, the calculator produces two modeling deltas in the operation-based format by implementing a specific DOL Interface: the active delta representing the current model by create operations (cf. Figure 3) and the differences delta which leads the older version from the latest. If the first version of a model is given, the calculator produces only the active delta without the difference delta. Because, there is not previous versions to compare and detect the difference delta. Assigning the persistent identifiers to delta operations is also done by the difference calculator while matching model elements. These assignments are persistent over all sequences of the modeling deltas.

\section{Delta Optimizer}

In some cases, modeling deltas might contain some useless operations. To increase efficiency by reducing useless operations in modeling deltas, optimization of the delta operations is required. Optimization helps to receive more optimal modeling deltas, eventually. The Delta Optimizer (Figure 11) basically receives modeling delta as input.

There might be a lot of useless operations or redundancies in modeling deltas. For instance, if a particular model element is created and later deleted in the same delta (this might happen in the case of collaborative modeling at runtime), these two operations are registered for one element where both have no affect.

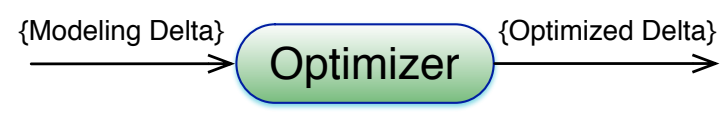

Figure 11: DOL-Service: Delta Optimizer

Another example might be changing one element several times in one modeling delta. In this case, it is optimal to save only the last change instead of several change operations for that model element.

Moreover, the order of delta operations in modeling deltas is also important to avoid the lost and fuzziness of change information while applying deltas to models by the patcher. The creation operations are lifted to the top and deletions are dropped to the end and changes are placed in the middle in each modeling delta. Creations are made firstly, then changes are made and deletions are made in the end. For instance, if a control flow has to be change to a newly created action node in the case of Activity diagrams, a new action node has to be created first, then an existing control flow can be reconnected to that new node.

After completing optimization process based on aforementioned criterion, the delta optimizer produces optimal modeling delta in the end. Besides, the delta optimizer can be utilized by other DOL-services to optimize the DOL-based modeling deltas. For example, the Patcher uses delta optimization to ease delta application process so that it can skip some delta operations between subsequent modeling deltas.

\section{Patcher}

The patcher allows to revert to earlier versions by applying (sequences of) modeling deltas to a model. For instance, modellers may need to roll back a model to an older version/state in the case of loss or damage of information. The inputs to the patcher are a model and a modeling delta. An input delta is applied to an input model and a result is previous model version (Figure 12). 


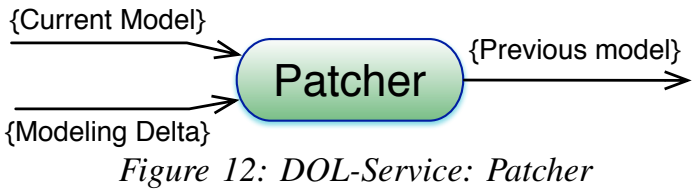

The delta operations are represented as executable descriptions in modeling deltas. For instance, execution of the active delta in the example in Section IV results in the current state of the model $\left(\operatorname{Ver}\right.$ sion $\left._{3}=\emptyset \operatorname{apply}\left(\Delta_{\text {active }}\right)\right)$. Executing each differences delta leads to the previous state from the current $\left(V e r\right.$ sion $_{2}=V$ ersion $\left.3 . \operatorname{apply}_{\left({ }^{3,2}\right)}\right)$ and Version $_{1}=$ Version $2 . \operatorname{apply}\left(\Delta_{(2,1)}\right)$. To apply differences deltas to the current model version, formerly the current model version has to be reverted from the active delta.

Considering that there are fifty versions of a model and the patcher is requested to revert the tenth version from the fiftieth. In this case, the patcher runs through a sequence of deltas between the fiftieth and the tenth versions and optimizes patching process by concatenating and optimizing the delta operations in these deltas. Several operations in these steps can be skipped using the optimizer to have faster and efficient reverting in the end.

\section{E. Change Tracer}

Being aware of changes on models is essential in comprehending and analysing the histories of evolving models. To this end, GMoVerS DOL provides the Change Tracer service which contributes the history analysis to reveal necessary information about the change history. The change tracer copes with extracting necessary information about whole evolutionary life-cycle of a model by focusing on a specific model element.

The change tracer receives the set of modeling deltas from the repository as input (as shown in Figure 13). Then, it seeks change information of a specific model element based on its persistent identifier by verifying the given set of modeling deltas. After all, detected information is reported as a set of connected correspondences so that it can be used in further analysis by visualizing.

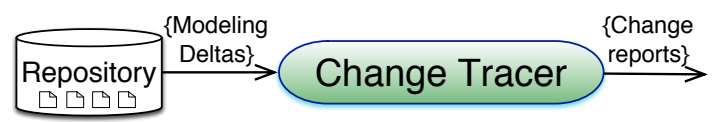

Figure 13: DOL-Service: Change Tracer

For instance, Figure 14 illustrates all history information of the control flow $g 4$ in the example in Section IV.

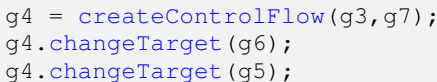

Figure 14: History information of Control Flow g4.

The traced element was referred to in three states. Because, it was in three different states in three model versions i.e it was changed subsequently. Information about these states is tracked by slicing three modeling deltas, the active delta in Figure 3 and two differences deltas in Figures 4 and 5.

\section{IMPLEMENTATION}

This section discusses the prototype implementation of the DOL-services integrated into the Eclipse environment. First of all, this section indicates which tools and techniques are required to derive a specific DOL for a specific modeling language and to implement all the DOL-services. Then, the selected tools and techniques are explained based on their role in the prototype implementations.

All model designing tools have their own internal model representation technique. Therefore, internal representations are required for both instance and meta-models. This allows the DOL-services to manipulate models being a part of the specific orchestration of any DOL-application.

To manipulate models within the DOL-application platforms, in-place model transformations are needed. Model manipulations help to apply changes to models and shift a model between states which it undergoes during the evolution process. The DOL-services like the Patcher and the Adapter take advantage of in-place model manipulations to fulfil their missions. The Difference Calculator requests the model traversal system to compare the given models and calculate the differences between them.

The requirements for the implementation tools and techniques are solved by JGraLab (Java Graph Laboratory) API [24]. First of all, JGraLab provides means to handle meta-models defining Graph Schema. Thereafter, TGraphs conforming the given graph-schema can be manipulated and handled by JGraLab API. To represent models internally, GMoVerS DOL uses TGraphs [19] that are accessible by JGraLab API. Vertices and edges are the first-class objects in TGraphs and they can be attributed, are directed and typed. JGraLab supports management of TGraphs, and in-place graph transformations such as creation, deletion and change of graph elements. Finally, JGraLab has its own graph querying system which allows to filter, traverse and to compare the query results.

The meta-model of a modeling language is designed in RSA [18] as UML class diagrams and loaded into JGraLab. When a meta-model is loaded, the DOL Generator automatically generates the DOL Interface. Likewise, the meta-model is used by the adapter to parse models in the exchange XMI format to TGraphs. The instance models are designed in RSA as well and parsed to TGraphs by the adapter. Both, metaand instance models are exported in the exchange formats without layout information. Eventually, all DOL-services like the calculator and the patcher directly operate on the TGraphbased models using JGraLab's in-place transformations and querying system.

The DOL-based difference representation approach is also implemented by VIATRA (VIsual Automated model TRAnsformations) in [25]. GMoVerS DOL is not strict only to JGraLab and can be realized by other model transformation and representation approaches such as QVT (Query/View/Transformation) [26]. But in some cases, model transformation approaches aim at transform from source model to target model lacking in-place manipulations which also makes implementations more complicated. For example, some experiments showed that deleting a model element is solved by an unspecified transformation rule in the case of ATL (ATLAS 
Transformation Language) [27] i.e. if a model element has to be deleted during transformation, a rule for that deletion is not specified so that an element supposed be deleted is not copied into a new model.

\section{APPLICATIONS}

In order to present applicability of the approach, several use cases are explained in this paper. These use cases are model versioning, model history analysis and collaborative modeling that are discussed in follow up sections in detail. The architectures of these applications are developed by the specific orchestrations of the DOL-services explained in Section VI and built on the underlying principles and properties listed in Section II.

\section{A. Model Versioning}

Model versioning aims at managing and manipulating models as well as storing and reusing the model differences. Therefore, version management systems are important to handle models and their histories. To this end, the Generic Model Versioning System (GMoVerS) is established adopting a specific orchestration of the DOL-services in this section.

The DOL-based difference representation approach is applied to versioning Sustainability Reports at companies in [28]. As sustainability reports at companies are also subject to constant changes and evolution, companies intend to report and analyse sustainability information to provide sustainable future. Thus, reports have to be versioned to analyse histories of sustainability reports and to present changes. To derive a specific DOL for representing differences of report versions, the approach employed a schema of sustainability reports.

The approach is applied to UML activity diagrams in this paper. In GMoVerS, the model differences are represented in terms of the DOL operations and the construction of version control activities is achieved by the amalgamation of the DOLservices as displayed in Figure 15.

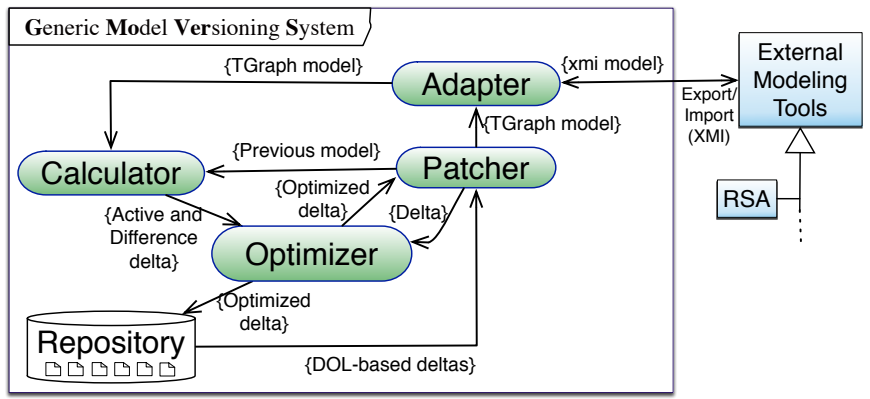

\section{Figure 15: Specific orchestration of the DOL-services for GMoVerS}

Current implementations of GMoVerS support adding models to the versioning system, committing changes and reverting older versions. In each of these services, relevant DOL-services are involved in a certain order based on dataflow.

For example, to start version control with a new model, a model has to be added under version control. It requires involvement of the adapter to parse a model in the exchange format to internal representation, the calculator to produce the active delta for the new model. Similarly, committing changes requires the adapter to parse new versions to internal representation, the patcher to revert the previous model version, again the calculator to calculate the differences between the previous and committed versions and produce the differences and active deltas. Reverting needs the patcher firstly to revert the recent model version, then it is involved as many times as needed to revert a requested version. For instance, to revert the first version form the third version, the patcher is called three time: one time for reverting the recent model from the active delta, two times to apply two the differences deltas to the current version.

Figure 16 displays a screen-shot of the GMoVerS development environment which is elaborated applying the DOLservices.

The package explorer on the left side shows the arrangement of the workspace including the meta-model in Figure 1 , the models and modeling deltas of the example in Section IV. Particularly, all DOL-based modeling deltas belong to the GMoVerS repository. On the upper row of the right side, Figure 16 displays three subsequent versions of the example model in Figure 2 in the exchange format. The central row of the right shows two differences deltas and the active delta in Figures 3,4 and 5. Finally, the most bottom of the screen-shot is a window to type and execute aforementioned activities such as add, commit and patch.

While committing local model changes to the main repository, conflicts might occur between differentiated models. In such cases, conflicts have to be detected when they arise and resolved either automatically (if possible) or semiautomatically. If the user involvement is needed in the case of semi-automatic way, version control system has to provide interactive conflict resolution feature by browsing conflicts. To this end, implementation of conflict resolver DOL-services is an ongoing work.

\section{B. Model History Analysis}

Analyzing the model histories is the best aid in comprehending and understanding what changes are made by designers or to know how a model evolves. Also, observing the model history and its evolution process assists the users in making important decisions about next life of model-based software projects.

The history analysis is built on the top of the change tracer (Figure 17). In order to detect history information of a specific model element, the change tracer fetches a set of modeling deltas from the repository and runs throughout these deltas based on a persistent identifier by gathering required information from each delta. After detecting necessary history information, it is visualized in a tabular view.

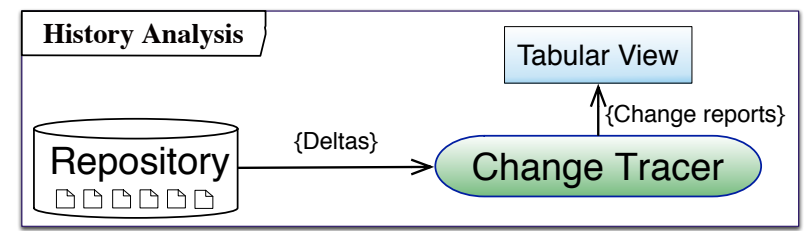

Figure 17: Specific orchestration of the DOL-services for history analysis 


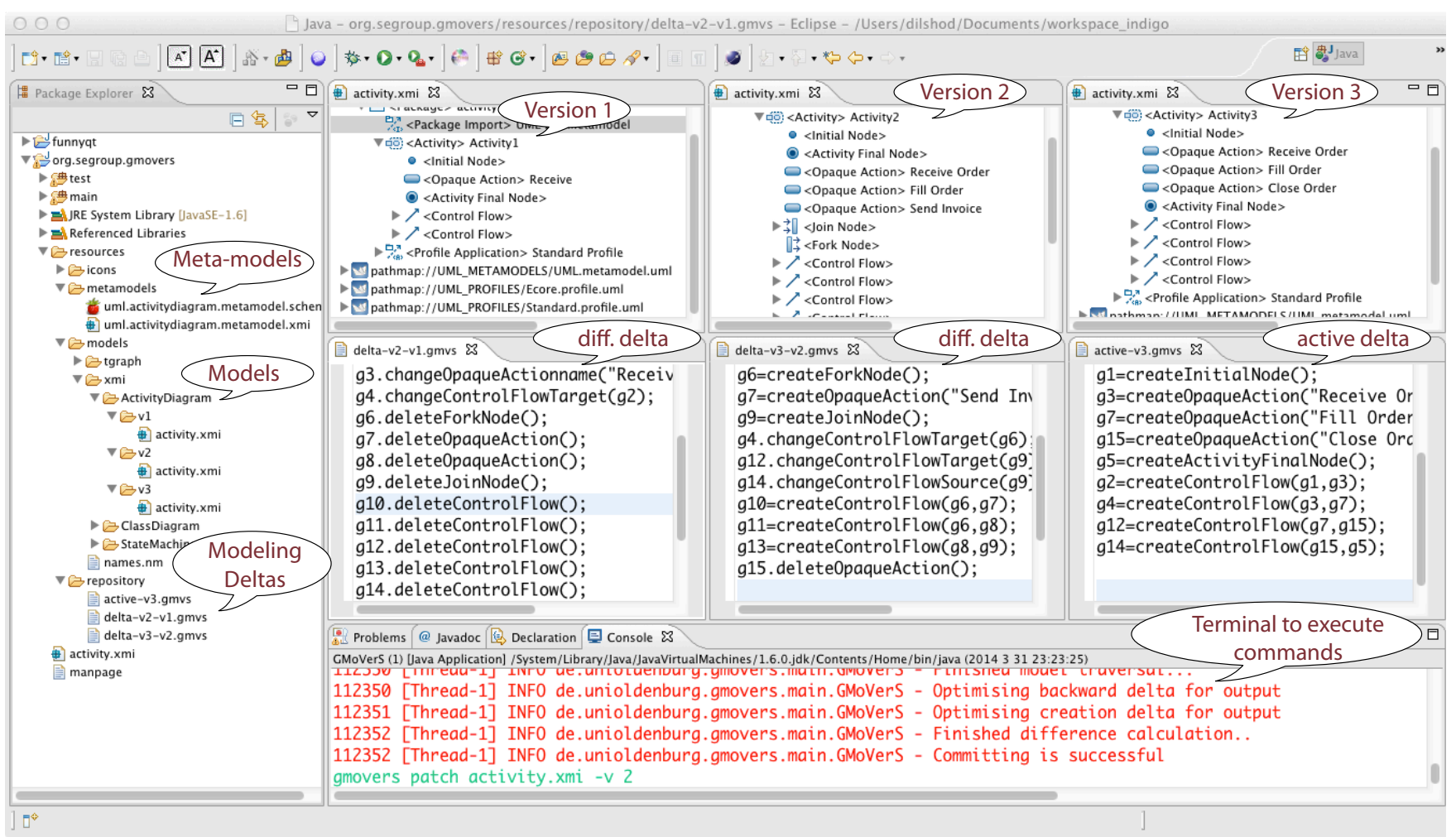

Figure 16: A screen-shot of GMoVerS

The screen-shot in Figure 18 displays the example model in Section IV. The user interface of the model history analyser has two sections: The Model Tree section shows model versions in a tree view including their history information and the tabular view on the right side lists the history information for a specific model element. To see the history information of any model element, it has to be selected from the model tree and click the Show History button. On the same line with the show history button, the label shows the name of a model element which is being traced. The history table contains three columns: Versions - the model version number which the change is made, Modification Type - the kind of the change and Date - the date which the change is made.

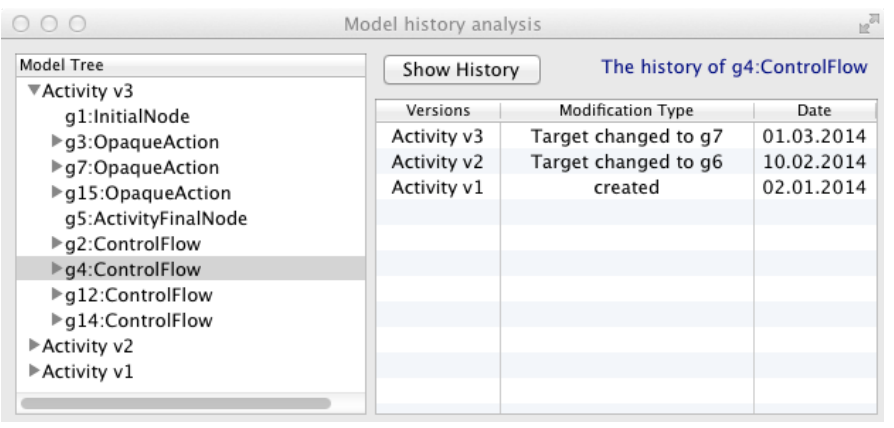

\section{Figure 18: A screen-shot of the history analysis application}

The history analysis application firstly builds a tree for each model version running throughout all modeling deltas to show models as a general tree. For example, on the left side of Figure 18 , all model versions are outlined in a tree view including all model elements with their attributes. Then, the change tracer service traces the history of selected model element based on its persisted identifier. The table on the right side shows the history of the g4:Control Flow which is selected from the left tree. The change tracer queries states of the selected element in each version and lists the detailed history of that element including the kind of change and associated objects.

\section{Collaborative Modeling}

Another prominent application of DOL is collaborative modeling which facilitates a teamwork of several designers on a shared model at runtime. Currently, the implementation of this application is planned as a students project. It is intended to deliver a collaborative modeling environment which is built on top of the DOL-based difference representation approach.

The implementation of the application will rely on representing changes (made by collaborators) in terms of the DOLoperations and exchanging these changes by the appropriate modeling deltas. To this end, it is planned to develop extra DOL-service named runtime operation recorder to detect operations embodying changes. The operation recorder will be integrated into end-user model designing tools and it detects changes made by modellers. While manipulating a model, the DOL-based change operations are recorded in modeling deltas. Since a model is manipulated by a group of modellers at runtime, an evolving model has several development branches. In this case, synchronization is required among various development branches. Synchronization of changes basically is synchronization of the DOL-based modeling deltas which stored in various workspaces. Synchronization of changes will be eased by exchanging small DOL-based deltas. Eventually, various model designing tools which are running on different platforms can communicate with each other in terms of delta operations language. 


\section{CONClusion AND Future Work}

This paper introduced the operation-based approach to model difference representation facilitating several additional services. DOL and its services satisfy all the requirements mentioned in Section II:

- Meta-model Generic. DOL is applicable to several modeling languages with respect to their meta-models. The DOL approach is applied to UML activity diagram in this paper, to versioning Sustainability Reports in [28] and the prototype experiments cover UML state machines.

- Tool Independent. The approach has its own internal model representations. But, the DOL-services provide an Adapter for integration with external modeling tools by the exchange format.

- Operation-based. A specific DOL is generated by the DOL generator from the meta-model of a modeling language. The model differences are represented in terms of the operation-based DOL embodying changes between subsequent model versions. Each DOL operation encloses all necessary information about a change, has a meaningful syntax and completely follows compound modeling concepts.

- Delta-based. Only changed model objects are referred in modeling deltas. Also, modeling deltas are the executable descriptions of the model differences which allow to form older versions of a model. These descriptions are implemented by in-place model transformations.

- Completeness. The DOL-based representation carries precise information about each change including the kind of change and the referenced modeling concept. The changed model objects are referred to according to their persistent identifiers stored in modeling deltas.

- Reusability. DOL provides several DOL-services which can directly access and manage the DOL-based modeling deltas. The DOL-services make modeling deltas straightforward and accessible fur further analysis and manipulations enabling applicability to various application areas.

A specific DOL is derived for a specific modeling language and several DOL-services are provided which can manage DOL-based deltas. Services relying on representation of the GMoVerS DOL approach are applied to model versioning and model history analysis so far, using state-based difference calculation. The DOL-services list will be extended with $a$ runtime operation recorder, a difference merger and a synchronizer for collaborative modeling.

\section{REFERENCES}

[1] Object Management Group, "XMI Specification, v1. 2."

[2] A. Cicchetti, "Difference Representation and Conflict." Ph.D. dissertation, University of L'Aquila, (Italy), April 2008.

[3] EMF: Eclipse Modeling Framework (Compare), http://www.eclipse.org/emf/compare.

[4] J. Loeliger, Version Control with Git: Powerful Tools and Techniques for Collaborative Software Development. O'Reilly Media, 2009.
[5] B. Collins-Sussman, B. W. Fitzpatrick, and C. M. Pilato, Version Control with Subversion. O'Reilly Media, 2004.

[6] W. F. Tichy, RCS - A System for Version Control. Software - Practice Experience. Volume 15, Issue 7, 1985.

[7] M. Alanen and I. Porres, "Difference and union of models." in $U M L$ 2003. LNCS. Springer, 2003, pp. 2-17.

[8] A. Cicchetti, D. Di Ruscio, and A. Pierantonio, "A metamodel independent approach to difference representation." Journal of Object Technology, vol. 6:9, pp. 165-185, October 2007.

[9] K. Altmanninger, A. Bergmayr, W. Schwinger, and G. Kotsis, "Semantically enhanced conflict detection between model versions in SMoVer by example." in Proceedings of the Int. Workshop on Semantic-Based Software Development at OOPSLA, 2007.

[10] A. Haber, K. Hölldobler, C. Kolassa, M. Look, B. Rumpe, K. Müller, and I. Schaefer, "Engineering Delta Modeling Languages." in Proceedings of the 17th International Software Product Line Conference. ACM, 2013, pp. 22-31.

[11] T. Kehrer, U. Kelter, and G. Taentzer, "A Rule-Based Approach to the Semantic Lifting of Model Differences in the Context of Model Versioning." in $A S E, 2011$, pp. 163-172.

[12] T. Kehrer, M. Rindt, P. Pietsch, and U. Kelter, "Generating Edit Operations for Profiled UML Models.” in ME@MoDELS, 2013, pp. 30-39.

[13] UML: Unified Modeling Language, http://www.uml.org.

[14] Mathworks: Matlab/Simulink, http://mathworks.com/simulink.

[15] J. Helming and M. Koegel, "EMFStore." 2013, http://eclipse.org/emfstore.

[16] S. Krusche and B. Bruegge, "Model-based Real-time Synchronization." in International Workshop on Comparison and Versioning of Software Models (CVSM'14), February 2014.

[17] Visual Paradigm, "Visual Paradigm for UML." UML tool for software application development, 2010.

[18] IBM Rational Software Architect, http://www.ibm.com.

[19] J. Ebert, V. Riediger, and A. Winter, "Graph Technology in Reverse Engineering, The TGraph Approach." in 10th Workshop Software Reengineering (WSR 2008), R. Gimnich, U. Kaiser, J. Quante, and A. Winter, Eds., vol. 126. GI (LNI), 2008, pp. 67-81.

[20] C. Küpker, "General Model Difference Calculation." Bachelor Thesis, Carl von Ossietzky University of Oldenburg, June 2013.

[21] Z. Xing and E. Stroulia, "UMLDiff: An Algorithm for Object-Oriented Design Differencing." ser. 6. ACM, 2005, pp. 54-65.

[22] M. Schmidt and T. Gloetzner, "Constructing Difference Tools for Models Using the SiDiff Framework." ICSE 2008, pp. 947-948, May 10-18 2008 .

[23] C. Treude, S. Berlik, S. Wenzel, and U. Kelter, "Difference Computation of Large Models." in Proceedings of the the 6th Joint Meeting of the European Software Engineering Conference. ACM Press, 2007, pp. 295-304.

[24] S. Kahle, "JGraLab: Konzeption." Entwurf und Implementierung einer Java-Klassenbibliothek für TGraphen, 2006.

[25] D. Varró and A. Balogh, "The Model Transformation Language of the VIATRA2 Framework." Sci. Comput. Program., vol. 68, no. 3, pp. 187-207, October 2007. [Online]. Available: http: //dx.doi.org/10.1016/j.scico.2007.05.004

[26] Object Management Group., "Meta object facility (mof) 2.0 query/view/transformation (QVT) specification." Final Adopted Specification (November 2005), 2008.

[27] J. Frédéric, A. Freddy, B. Jean, K. Ivan, and V. Patrick, "ATL: a QVTlike transformation language." in Companion to the 21st ACM SIGPLAN symposium on Object-oriented programming systems, languages, and applications. ACM, 2006, pp. 719-720.

[28] D. Kuryazov, A. Solsbach, and A. Winter, "Versioning Sustainability Reports." in 5.BUIS-Tage: IT-gestütztes Ressourcen- und Energiemanagement. Springer-Verlag, 2013, pp. 409-419. 